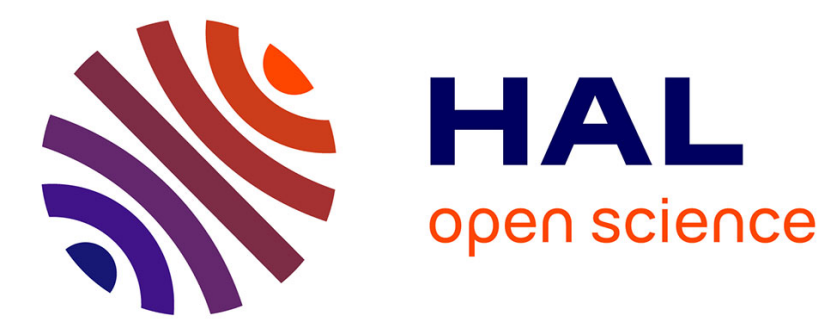

\title{
François, pape des migrants ?
}

Vincent Geisser

\section{To cite this version:}

Vincent Geisser. François, pape des migrants ?. Migrations Société, 2017, 167 (1), pp.3. 10.3917/migra.167.0003 . halshs-02113025

\section{HAL Id: halshs-02113025 https://shs.hal.science/halshs-02113025}

Submitted on 30 Apr 2019

HAL is a multi-disciplinary open access archive for the deposit and dissemination of scientific research documents, whether they are published or not. The documents may come from teaching and research institutions in France or abroad, or from public or private research centers.
L'archive ouverte pluridisciplinaire HAL, est destinée au dépôt et à la diffusion de documents scientifiques de niveau recherche, publiés ou non, émanant des établissements d'enseignement et de recherche français ou étrangers, des laboratoires publics ou privés. 


\section{Editorial}

\section{FRANÇOIS, PAPE DES MIGRANTS ?}

\section{Vincent Geisser}

Centre d'information et d'études sur les migrations internationales | «Migrations Société »

2017/1 N 167 | pages 3 à 12

ISSN 0995-7367

Article disponible en ligne à l'adresse :

https://www.cairn.info/revue-migrations-societe-2017-1-page-3.htm

Distribution électronique Cairn.info pour Centre d'information et d'études sur les migrations internationales.

(C) Centre d'information et d'études sur les migrations internationales. Tous droits réservés pour tous pays.

La reproduction ou représentation de cet article, notamment par photocopie, n'est autorisée que dans les limites des conditions générales d'utilisation du site ou, le cas échéant, des conditions générales de la licence souscrite par votre établissement. Toute autre reproduction ou représentation, en tout ou partie, sous quelque forme et de quelque manière que ce soit, est interdite sauf accord préalable et écrit de l'éditeur, en dehors des cas prévus par la législation en vigueur en France. Il est précisé que son stockage dans une base de données est également interdit. 


\section{François, pape des migrants?}

Vincent GEISSER

"Chers frères et soeurs migrants et réfugiés! À la racine de l'Évangile de la miséricorde, la rencontre et l'accueil de l'autre se relient à la rencontre et à l'accueil de Dieu : accueillir l'autre, c'est accueillir Dieu en personne!».

Pape François ${ }^{\mathrm{I}}$

"Le monde que décrit François est une chimère, une illusion, un univers rose bonbon, gentillet, bondé de clandestins bisounours. François vit dans un rêve parallèle à la réalité crue! ».

Francesca de Villasmundo²

« François n'est pas un jésuite pour rien... et s'il reprend le discours immigrationniste de son prédécesseur, c'est pour mieux l'enrober de la doucereuse guimauve des bons sentiments et de la parole chrétienne dont il sait combien elle va annihiler celle de ses ouailles tentées d'ouvrir les yeux sur une réalité qui les accable chaque jour davantage ».

Robert Albarèdes 3 .

$\mathrm{F}_{\mathrm{n}}$ janvier 20I7, les Éditions de l'Atelier publiaient un ouvrage au titre évocateur Ce pape qui dérange 4 , rédigé par Virginie Riva, correspondante à Rome pour la radio Europe i. Il est vrai que les livres consacrés au souverain pontife se suivent et se ressemblent, oscillant généralement entre registre hagiographique et registre critique, les auteurs jouant de leur proximité avec la curie romaine pour livrer aux lecteurs toutes sortes d'informations et de confidences inédites - qui le sont rarement en réalité - sur les « secrets du Vatican » et l'intimité du pape. Or, dans l'ouvrage de Virginie Riva, on ne trouve rien de cela. Bien que journaliste, l'auteure s'est moins intéressée aux « intrigues vaticanesques » qu'aux discours et aux projets sociaux de François, qu'elle présente

1. PAPE FRANÇOIS, Message pour la Journée mondiale des migrants et des réfugiés [En ligne], 17 janvier 2016, https://w2.vatican.va/content/francesco/fr/messages/migration/documents/papafrancesco_20150912_world-migrants-day-2016.html.

2. DE VILLASMUNDO, Francesca, "Le pape s'en prend à nouveau à l'Europe à propos des migrants [En ligne]", Medias-presse.info, 28 février 2017, http://www.medias-presse.info/lepape-sen-prend-a-nouveau-a-leurope-a-propos-des-migrants/70294/.

3. ALBARÈDES, Robert, "Le Pape François ? Un bon soldat de la mondialisation immigrationniste [En ligne]", Riposte laïque, 19 juin 2014, https://ripostelaique.com/le-pape-francoisun-bon-soldat-de-la-mondialisation-immigrationniste.html.

4. RIVA, Virginie, Ce pape qui dérange, Ivry-sur-Seine : Éditions de L’Atelier, 2017, 173 p. 
comme un véritable programme politique d'un pape à la fois chef de l'Église mais aussi chef d'État, qui recourt ainsi à la « méthode synodale » pour faire bouger les lignes. Car, au regard de ses premières décisions et actions, il apparait clairement que le premier pape latino-américain de l'histoire de l'Église souhaite rompre avec les modes de gouvernance de ses prédécesseurs, très fortement marqués par un eurocentrisme provincial et petit-bourgeois, en accordant davantage d'attention à ce qu'il qualifie lui-même de "périphéries humaines et sociales" ${ }^{5}$. Depuis son élection en mars 2013, le pape François a ouvert de nombreux chantiers, comme la critique des ravages de la mondialisation, l'homosexualité, la lutte contre la corruption et les réseaux mafieux, le statut des femmes au sein de l'Église, le problème de la pédophilie des clercs, la redéfinition d'une nouvelle diplomatie vaticane, etc., qui viennent bousculer à la fois les esprits conservateurs et les routines de fonctionnement de l'institution ecclésiale. Ce n'est probablement pas un hasard si ces projets de réforme suscitent aujourd'hui autant d'oppositions et de résistances au sein même de la curie romaine: "L'ensemble des chantiers entamés par le pape dessine une exigence, issue de sa prière, de sa formation jésuite, de sa théologie sudaméricaine... Et c'est bien cette exigence qui dérange. C'est celle d'un catholicisme qui implique un comportement tout à fait nouveau, une attitude beaucoup plus profonde que la simple adhésion aux dogmes, un intérêt porté aux pauvres plutôt qu'un respect strict des valeurs non négociables prônées pendant des décennies par Jean-Paul II puis Benoit XVI $»^{6}$.

Et s'il y a un domaine où le discours du pape dérange plus que tout autre, c'est probablement celui de l'immigration, où l'Église est souvent restée confinée au registre de l'assistance et de la charité, sans véritablement s'attaquer au fond du problème. De ce point de vue, le pape François paraît renouer avec la philosophie humaniste de Giovanni Battista Scalabrini $(1839-1905)^{7}$, fondateur de l'ordre catholique des Scalabriniens ${ }^{8}$, qui consacra une large partie de son action pastorale aux migrants. Comme nous le rappelions dans un précédent éditorial, «l'évêque italien était en avance sur de nombreux penseurs socialistes et progressistes de son temps. Son interprétation du fait migratoire, pourtant développée à la fin $d u X I X^{e}$ siècle, reste d'une surprenante actualité, anticipant largement les phénomènes de mondialisation et les processus complexes d'intégration des migrants au sein des sociétés des différents pays d'accueil »?. À l'instar de

5. Ibidem (voir p. 8).

6. Ibidem (voir p. 9).

7. PEROTTI, Antonio (sous la direction de), L'Église et les migrations. Un précurseur : Giovanni Battista Scalabrini, Paris : Éd. CIEMI-L'Harmattan, 1997, 224 p.

8. Le Centre d'information et d'études sur les migrations internationales (CIEMI), qui édite la revue Migrations Société, a été fondé en 1977 par des prêtes catholiques appartenant à la Congrégation des missionnaires de Saint-Charles Borromée-Scalabriniens.

9. GEISSER, Vincent, "Immigration, nationalité, solidarité : I'Église a-t-elle encore quelque chose à dire ?", Migrations Société, vol. 25, n 145, janvier-février 2013, pp. 7-16. 
Giovanni Battista Scalabrini, le pape François voit dans le migrant l'incarnation par excellence de la figure du pauvre: "Dans le monde actuel, aux portes d'une Europe bien portante, le pauvre et l'indifférence dont il est victime s'incarnent aussi pour le pape dans la figure du migrant $\aleph^{{ }^{\circ}}$. Sa sensibilité à l'égard de la question migratoire n'est pas nouvelle et doit pour beaucoup à son héritage familial et à sa formation jésuite. Le pape François aime à rappeler qu'il est lui-même un enfant d'immigré italien venu du Piémont : son père, Mario José Bergoglio, est arrivé en 1927 en Argentine et a failli périr en mer. Sa filiation migrante a d'ailleurs marqué toute sa trajectoire en tant que clerc de l'Église, d'abord comme séminariste, puis comme simple prêtre, et enfin comme évêque et cardinal de Buenos Aires, capitale argentine, où il a développé de nombreuses actions pastorales dans les quartiers populaires et les bidonvilles peuplés de migrants en provenance des autres pays d'Amérique latine (Paraguay, Bolivie, Pérou, etc.). Bousculant le cérémonial et le protocole dus à son rang, Jorge Mario Bergoglio avait l'habitude de se rendre en transports en commun dans les faubourgs de Buenos Aires pour célébrer des messes, baptiser des enfants, et rencontrer des familles déshéritées. François a aussi été fortement influencé par les enseignements du père jésuite Pedro Arrupe qui, en 1974, publia un décret demandant aux membres de la Congrégation de Jésus d'acquérir une expérience personnelle de la pauvreté. En I980, le père Pedro Arrupe fonda le Service jésuite des réfugiés, actuellement présent dans cinquante pays et qui fournit de l'aide à près d'un million de personnes ${ }^{\mathrm{II}}$.

Compte tenu de son passé familial et de son expérience sacerdotale, il n'est donc pas étonnant que François ait placé la question migratoire au cœur de son «programme pontifical». Ainsi, dès sa première lettre d'exhortation apostolique (Evangelii gaudium : la joie de l'Évangile) datée du 24 novembre 2013, il consacre plusieurs lignes aux migrants, mettant en garde les croyants contre les réflexes de peur et de rejet : «Les migrants me posent un défi particulier parce que je suis Pasteur d'une Église sans frontières qui se sent mère de tous. Par conséquent, j'exhorte les pays à une généreuse ouverture, qui, au lieu de craindre la destruction de l'identité locale, soit capable de créer de nouvelles synthèses culturelles. Comme elles sont belles les villes qui dépassent la méfiance malsaine et intègrent ceux qui sont différents, et qui font de cette intégration un nouveau facteur de développement! Comme elles sont belles les villes qui, même dans leur architecture, sont remplies d'espaces qui regroupent, mettent en relation et favorisent la reconnaissance de l'autre! ${ }^{12}$.

10. RIVA, Virginie, Ce pape qui dérange, op.cit. (voir p. 102).

11. CALVEZ, Jean-Yves, "Les choix du père Arrupe", Études, $n^{\circ} 4$, tome 407, octobre 2007, pp. 355-365.

12. PAPE FRANÇOIS, "La joie de l'évangile. Exhortation apostolique du 24 novembre 2013", La Documentation Catholique, $\mathrm{n}^{\circ} 2513$, janvier 2014, pp. 6-83. 
ais c'est surtout par des gestes symboliques forts que le nouveau pape entend faire connaître au monde en général, et aux élites dirigeantes en particulier, sa «théologie de l'accueil et de l'altérité », n'hésitant pas à s'en prendre directe ment aux politiques sécuritaires et aux discours populistes qui instrumentalisent la peur de l'Autre.

Contre l'avis de la diplomatie vaticane ${ }^{\mathrm{r} 3}$, il décide d'effectuer son premier déplacement apostolique sur l'île de Lampedusa, le 8 juillet 2013. À cette occasion, il prononce une homélie dans laquelle il dénonce avec force «la mondialisation de l'indifférence », visant indirectement les politiques sécuritaires de l'Union européenne (UE) en mer Méditerranée qui aboutissent aux décès et aux disparitions de plusieurs milliers de migrants : «Dans ce monde de la mondialisation, nous sommes tombés dans la mondialisation de l'indifférence. Nous sommes habitués à la souffrance de l'autre, cela ne nous regarde pas, ne nous intéresse pas, ce n'est pas notre affaire! [...] "Adam où estu?", "Où est ton frère?", sont les deux questions que Dieu pose au début de l'histoire de l'humanité et qu'il adresse aussi à tous les hommes de notre temps, à nous aussi. Mais je voudrais que nous nous posions une troisième question : Qui de nous a pleuré pour ce fait et pour les faits comme celui-ci? Qui a pleuré pour la mort de ces frères et soeurs? Qui a pleuré pour ces personnes qui étaient sur le bateau? Pour les jeunes mamans qui portaient leurs enfants? Pour ces hommes qui désiraient quelque chose pour soutenir leurs propres familles? Nous sommes une société qui a oublié l'expérience des pleurs, du "souffrir avec" : la mondialisation de l'indifférence nous a ôté la capacité de pleurer! $»^{14}$.

En février 20I6, François choisit symboliquement comme dernière étape de son voyage pontifical au Mexique, la ville frontalière de Ciudad Juárez, particulièrement stigmatisée par le voisin états-unien en raison de la présence de filières d'immigration clandestine. Il y célèbre une messe devant 200000 fidèles à proximité de la clôture métallique qui sépare le Mexique des ÉtatsUnis. À cette occasion, il appelle à «construire des ponts» et «non des murs», répondant indirectement au discours démagogique du candidat républicain Donald Trump, alors en tête des sondages avant les primaires de son parti, et qui incite dans ses meetings électoraux à combattre les immigrés mexicains assimilés à des «criminels» et à des «violeurs». D'ailleurs, quelques mois auparavant, en septembre 20I5, devant le Congrès américain, le pape François avait clairement appelé les représentants et les sénateurs à ne pas tourner le dos à leur histoire et leur mémoire migratoires: «Nous, le peuple de ce continent, nous n'avons pas peur des étrangers, parce que la plupart d'entre nous étaient autrefois des étrangers. Je vous le dis en tant que fils dimmigrés, sachant que beaucoup d'entre vous sont aussi des descendants d'immigrés $»^{15}$.

13. MOUNIER, Frédéric, "Le pape François fustige 'l'indifférence'", La Croix, 8 juillet 2013.

14. PAPE FRANÇOIS, "'Où est ton frère ?'. Une question adressée à chacun", La Documentation Catholique, $\mathrm{n}^{\circ} 2512$, octobre 2013, pp. 110-111.

15. MAILLARD, Sébastien, "Au Congrès, le pape invite les Américains à rester dignes de leur rêve" La Croix, 24 septembre 2015. 
À l'occasion de la cérémonie du Jeudi saint, le 24 mars 20I6, le pape fait le choix de laver les pieds de onze réfugiés résidant dans un foyer de la banlieue de Rome, rappelant le geste accompli par Jésus pour ses douze apôtres. Si cet acte symbolique a été plutôt apprécié dans les milieux « progressistes » de l'Église, les cercles majoritairement conservateurs et traditionnalistes l'ont perçu comme une forme de provocation papale, voire comme une menace pour la catholicité, et ce d'autant plus que parmi les migrants choisis trois étaient de religion musulmane : "Jour solennel de la vie liturgique de l'Église catholique, le Jeudi saint est devenu, sous l'ère bergoglienne, et grâce à la complicité de médias complaisants, un manifeste socialisant et gauchiste, où immigration, injustice sociale, féminisme, ont la part belle. Tous les moyens sont bons, même les plus sacrés, pour révolutionner et réformer l'humanité à travers le prisme d'une idéologie utopiste et chimérique, progressiste et folle, d'une société déterminée par les concepts de liberté, égalité et fraternité. Idéologie qui envoûte le pape argentin $»^{16}$.

Mais de toutes les initiatives pontificales, c'est surtout le déplacement du pape sur l'île grecque de Lesbos, le I6 avril 20I6, qui a été le plus commenté et source de controverses publiques. Depuis que l'Union européenne a décidé de fermer ses frontières, plus de 50 ooo migrants sont bloqués en Grèce, transformant les îles en véritable prison à ciel ouvert. De plus, l'accord entre l'ue et la Turquie du I8 mars $2016^{17}$ a contribué à rendre la situation davantage dramatique : les centres de tri, censés être temporaires, sont devenus progressivement des camps fermés, où des milliers de réfugiés s'entassent. Au-delà du registre strictement humanitaire, la présence du pape François à Lesbos revêt donc une signification politique, remettant en cause le bien-fondé de la politique restrictive et sécuritaire de l'Europe. Pour le souverain pontife, certes, la peur du migrant est compréhensible dans des sociétés européennes confrontées à une profonde crise économique et sociale mais, elle ne saurait justifier les traitements inhumains, surtout quand ils touchent des individus vulnérables fuyant des situations de guerre (enfants, femmes et vieillards) : " Je ne sais pas. Je comprends les gouvernements, ainsi que les peuples, qui ont une certaine peur. Cela je le comprends, et nous devons faire preuve d'une grande responsabilité dans l'accueil. Un des aspects de cette responsabilité est ceci: comment pouvons-nous intégrer ces gens et nous. J'ai toujours dit qu'ériger des murs n'est pas une solution: nous en avons vu tomber un, au siècle dernier. Cela ne résout rien. Nous devons faire des ponts. Mais les ponts se font avec

16. DE VILLASMUNDO, Francesca, "Une Pâques aux couleurs des immigrés musulmans : le pape François leur lavera les pieds le Jeudi Saint" [En ligne], Medias-Press.info, 15 mars 2016, http://www.medias-presse.info/une-paques-aux-couleurs-des-immigres-le-pape-francoisleur-lavera-les-pieds-le-jeudi-saint/51108/.

17. DE MARCILLY, Charles, "L'accord UE-Turquie et ses implications. Un partenariat incontournable mais sous conditions" [En ligne], Questions d'Europe, $\mathrm{n}^{\circ} 396,19$ juin 2016, http://www.robert-schuman.eu/fr/questions-d-europe/0396-I-accord-ue-turquie-et-sesimplicationsun-partenariat-incontournable-mais-sous-conditions. 
intelligence, ils se font par le dialogue, par l'intégration. Et pour cela, je comprends une certaine crainte. Mais fermer les frontières ne résout rien, parce que cette fermeture à la longue fait mal au peuple lui-même. L'Europe doit faire d'urgence des politiques d'accueil et d'intégration, de croissance, de travail, de réforme de l'économie... Toutes ces choses sont les ponts qui nous conduiront à ne pas ériger de murs $\aleph^{18}$.

Ce n'est pas tant la présence du pape à Lesbos qui a fait polémique, que l'initiative connexe, inspirée par la communauté de Sant' Egidio, coutumière des opérations de médiation et de maintien de la paix, de ramener avec lui, dans son avion personnel, douze réfugiés ${ }^{19}$. Le fait que les individus sélectionnés étaient tous musulmans, avec parmi eux des femmes portant le foulard islamique, a été considéré par certains milieux conservateurs de l'Église comme une véritable provocation, une atteinte à l'identité catholique (comment expliquer l'absence de réfugiés chrétiens parmi les "sélectionnés" dans un contexte où les Chrétiens d'Orient font l'objet de multiples persécutions ?), le pape François se voyant doublement accusé d'être « immigrationniste » et « islamophile». Le souverain pontife a d'ailleurs été contraint de se justifier publiquement, au risque de provoquer une crise de confiance au sein de sa propre famille catholique: "Je n'ai pas choisi entre musulmans et chrétiens. Ces trois familles avaient des documents prêts, en règle. Il y avait, dans une première liste, deux familles chrétiennes mais elles n'avaient pas les papiers en règle. Ce n'est donc pas un privilège. Ces douze sont tous des enfants de Dieu et je privilégie les enfants de Dieu! ${ }^{20}$.

S'il est vrai que les positions du nouveau pape dérangent certains Shiérarques de la curie romaine et les milieux les plus conservateurs de l'Église, il serait pourtant réducteur d'interpréter sa politique comme une rupture radicale avec les positions traditionnelles du Vatican sur les questions migratoires et le dialogue avec les croyants des autres religions $^{21}$. De son expérience de travail auprès des migrants dans les banlieues populaires de Buenos Aires, François en tire plutôt un certain pragmatisme, conciliant le respect de l'identité de l'Autre et l'impératif de cohésion sociale. Ce n'est donc pas par pur opportunisme que le pape François a récemment infléchi son discours dans le sens de l'intégration mais par souci d'exprimer plus clairement son point de vue, source de

18. PAPE FRANCOIS, Conférence de presse du Saint-Père au cours du vol de retour de Lesbos [En ligne], 16 avril 2016, https://w2.vatican.va/content/francesco/fr/speeches/2016/april/documents/papafrancesco_20160416_lesvos-volo-ritorno.html.

19. Sur les détails de l'affaire, voir RIVA, Virginie, Ce pape qui dérange, op. cit. (voir pp. 102-104).

20. Propos cités par GUÉNOIS, Jean-Marie, "Pourquoi le Pape est rentrée de Grèce avec des réfugiés musulmans" [En ligne], Le Figaro, 16 avril 2016, http://www.lefigaro.fr/actualitefrance/2016/04/16/01016-20160416ARTFIG00132-pourquoi-le-pape-est-rentre-de-greceavec-douze-refugies-musulmans.php.

21. GEISSER, Vincent, "Immigration, nationalité, solidarité : I'Église a-t-elle encore quelque chose à dire?", art. cité. 
nombreuses incompréhensions au sein de l'Église. Sur ce plan, le pape n'a rien d'un militant forcené du multiculturalisme et encore moins d'un partisan du relativisme culturel, il reste un intégrationniste convaincu, persuadé que les migrants doivent se fondre dans la culture des sociétés d'accueil et respecter leurs valeurs. L'effort doit se produire dans les deux sens : «Le phénomène migratoire pose donc un sérieux problème culturel, auquel on ne peut se dispenser de répondre. L'accueil peut donc être une occasion propice pour une nouvelle compréhension et ouverture d'horizon, tant pour celui qui est accueilli, lequel a le devoir de respecter les valeurs, les traditions et les lois de la communauté qui l'héberge, que pour cette dernière, appelée à valoriser tout ce que chaque immigré peut offrir à l'avantage de toute la communauté ${ }^{22}$.

Le pape ne verse jamais dans l'angélisme : il développe un regard profondément inquiet sur l'avenir du monde en général, et des sociétés européennes en particulier, marquées par les replis identitaires de toutes sortes et la montée des courants nationalistes et xénophobes. Mais de cette inquiétude il tire paradoxalement une forme d'espérance universelle, qu'il entend décliner sous le registre d'un «nouvel humanisme ». Pour François, l'Église ne pourra reconquérir sa centralité dans les sociétés en crise de repères et de valeurs, que si elle parvient à renouer avec la langue universelle du Christ, compréhensible par tous, y compris des incroyants et des non chrétiens : "Avec l'esprit et avec le cour, avec espérance et sans vaine nostalgie, comme un fils qui retrouve dans la mère Europe ses racines de vie et de foi, je rêve d'un nouvel humanisme européen, d'un chemin constant d'humanisation', requérant 'la mémoire, du courage, une utopie saine et humaine". Je rêve d'une Europe jeune, capable d'être encore mère : une mère qui ait de la vie, parce qu'elle respecte la vie et offre l'espérance de vie. Je rêve d'une Europe qui prend soin de l'enfant, qui secourt comme un frère le pauvre et celui qui arrive en recherche d'accueil parce qu'il n'a plus rien et demande un refuge. Je rêve d'une Europe qui écoute et valorise les personnes malades et âgées, pour qu'elles ne soient pas réduites à des objets de rejet improductifs. Je rêve d'une Europe où être migrant ne soit pas un délit mais plutôt une invitation à un plus grand engagement dans la dignité de l'être humain tout entier $\gg^{23}$.

Pour François, ce «nouvel humanisme » ne doit pas être seulement théorique. Il suppose un engagement concret de tous les catholiques quel que soit leur rang dans l'Église : "L'influence sur les politiques est importante, et la vigilance sur la manière dont elles sont appliquées, aussi. Mais les catholiques ne peuvent rester dans les idées : ils doivent agir et être des com-

22. PAPE FRANÇOIS, "Les migrations sont un élément fondamental de l'avenir du monde", La Documentation Catholique, $\mathrm{n}^{\circ}$ 2522, avril 2016, pp. 112-119.

23. PAPE FRANÇOIS, "Que t'est-il arrivé, Europe humaniste?", La Documentation Catholique, $n^{\circ} 2523$, juillet 2016, pp. 113-118. 
munautés accueillantes qui montrent qu'on peut s'engager, accueillir et intégrer. Les communautés chrétiennes peuvent être des lieux d'espoir ${ }^{24}$.

Ce message du pape a-t-il une chance d'être entendu par les catholiques français? Ces derniers sont-ils réceptifs au «nouvel humanisme » promu par le chef de l'Église?

Au sein de l'univers catholique français, les résistances sont nombreuses. Au même moment où était publié l'ouvrage de Virginie Riva, Ce pape qui dérange, un autre livre sortait en librairie : Église et immigration: le grand malaise. Le pape et le suicide de la civilisation européenne $e^{25}$. Le titre parle de lui-même. Son auteur, Laurent Dandrieu, essayiste et journaliste conservateur - il écrit notamment pour Valeurs Actuelles -, prétend porter la parole de la majorité catholique silencieuse, opposée aux orientations «pro-immigration» du nouveau pape. Sa critique du «nouvel humanisme » promu par François est virulente : « Ce livre est évidemment l'expression de mon propre malaise de fidèle catholique devant un discours ecclésial qui semble placer les catholiques devant un dilemme impossible: choisir entre la fidélité à l'Évangile qui, si l'on en croit le discours de l'Église depuis quelques décennies, nous obligerait à accueillir sans aucune réserve les migrants qui désirent venir en Europe, et la fidélité à notre patrie et à notre identité, que nous devrions sacrifier gaiement à cette absolutisation de l'accueil. Je l'ai donc écrit pour aider les catholiques à ne plus se sentir prisonniers de ce dilemme qui les condamne, soit à sembler renier les principes de leur foi, soit à consentir à leur propre disparition par fidélité à ces principes. C'est bien parce que cet accueil inconditionnel me semble suicidaire, et qu'il me paraît impossible que l'Église demande réellement à ses fidèles de renoncer à la défense de leur patrie, que ce discours de l'Église ne me paraît plus tenable et qu'elle doit impérativement le rectifier ${ }^{26}$.

Chez les catholiques ordinaires, le «nouvel humanisme » du pape François est accueilli de manière contrastée selon le degré des pratiques religieuses, les milieux socioprofessionnels, les contextes locaux et les orientations politiques. Déjà dans les années 1990-2000, le sociologue Jean-Luc Richard soulignait que les catholiques français étaient loin de former un groupe homogène, et qu'ils affichaient des positions diverses sur les questions de société en général, et sur la question migratoire en particulier : «Les catholiques de France ne constituent pas un groupe homogène qui s'exprimerait d'une seule voix sur la plupart des sujets sociaux d'importance, au nombre desquels

24. SENĖZE, Nicolas, "'Le pape François appelle à accueillir mais aussi à intégrer les migrants." Entretien avec Michael Czerny", La Croix, 3 février 2017.

25. DANDRIEU, Laurent, Église et immigration: le grand malaise. Le pape et le suicide de la civilisation européenne, Paris : Presses de la Renaissance, 2017, 288 p.

26. "'Pour en finir avec le messianisme de l'Église.' Entretien avec Laurent Dandrieu" [En ligne], Breizh-info.com, 21 janvier 2017, http://www.breizh-info.com/2017/01/21/60027/laurentdandrieu-finir-messianisme-migratoire-de-leglise-interview. 
l'immigration étrangère en France et ses conséquences ${ }^{27}$. Malgré cette hétérogénéité des opinions, Jean-Luc Richard faisait état d'une relative hostilité des catholiques français vis-à-vis de l'augmentation de l'immigration étrangère dans l'Hexagone bien que ne se traduisant cependant pas par un vote systématique en faveur du Front national. L'intégration objective à l'univers catholique (le fait d'aller à la messe, de militer dans une association chrétienne et de participer à des réseaux caritatifs, etc.) constituait souvent un frein au vote extrémiste. En ce sens, les catholiques français pouvaient émettre des opinions négatives sur l'immigration, tout en refusant de cautionner le discours radical de l'extrême droite. Ces dernières années, cette ambivalence des catholiques français à l'égard du fait migratoire semble persister. Plusieurs enquêtes confirment que plus d'un tiers des catholiques pratiquants et près de la moitié des catholiques non pratiquants restent réfractaires à l'accueil de nouveaux immigrés sur le territoire français. Toutefois, l'univers catholique français ne paraît pas être totalement insensible aux différents appels du pape. Plusieurs enseignements peuvent être tirés des résultats issus d'enquêtes récentes. D'abord, plus un individu est intégré à l'institution catholique, plus il se montrera réceptif au message du souverain pontife, notamment sur les questions sociales et migratoires. Ensuite, les catholiques restent traversés par des clivages politiques qui déterminent très largement leur position à l'égard de l'immigration. Sur ce plan, les dernières enquêtes tendraient à montrer que le tabou du vote d'extrême droite est désormais rompu dans l'univers catholique français. Le fait de se dire «catholique » - même "pratiquant» - ne préserve plus d'un vote en faveur du Front national (FN). Enfin, et c'est peut-être l'enseignement le plus important, les catholiques pratiquants ne semblent pas totalement sourds aux appels du pape à développer des actions concrètes en soutien pour les migrants : selon une enquête de l'Institut français d'opinion publique (IFOP), I4 \% des pratiquants ont déjà fait des dons en nature en faveur des migrants, $8 \%$ en argent et $24 \%$ ont l'intention de le faire. De même, $26 \%$ des catholiques pratiquants se disent prêts à donner de leur temps, voire à accueillir des migrants chez eux $^{28}$. Ces tendances sont d'ailleurs confirmées par une enquête plus récente : bien que traversés comme le reste des Français par des tendances anxiogènes à l'égard du fait migratoire et du fait musulman, les catholiques pratiquants (54\%), davantage que les non-pratiquants $(37 \%)$, seraient favorables à l'accueil sur le territoire européen des migrants débarquant sur les côtes italiennes et grecques ${ }^{29}$.

27. RICHARD, Jean-Luc, "Les catholiques, l'immigration étrangère et les tentations racistes en France. Quelques apports d'enquêtes d'opinion et de données macro-sociales", Migrations Société, vol. 24, n 139, janvier-février 2012, pp. 253-266.

28. INSTITUT FRANÇAIS D'OPINION PUBLIQUE, Les catholiques et l'accueil des migrants [En ligne], 24 septembre 2015, http://www.pelerin.com/Alaune/Migrantsledefipolitiquelesreponsespratiques/Sondagelescatholiquesetlaccueildesmigrants.

29. INSTITUT FRANÇAIS D'OPINION PUBLIQUE, Les catholiques, les Français et l'accueil des migrants [En ligne], avril 2016, 11 p., http://www.ifop.fr/media/poll/3377-1-study_file.pdf. 
Toutefois, il convient de ne pas se méprendre sur ces résultats et de conclure trop vite à la conversion massive des catholiques français et européens au «nouvel humanisme » prêché par François. Car, outre qu'il ne se dégage pas une majorité très forte chez les catholiques français pour soutenir ses appels à la solidarité en direction des migrants et des réfugiés, on peut penser qu'il s'agit davantage de l'expression d'une charité chrétienne très conformiste que le signe d'un ralliement puissant à la critique pontificale de la «mondialisation de l'indifférence ». En ce sens, la majorité des catholiques français, pratiquants et non pratiquants, s'inscrivent plus dans une logique humanitaire et charitable que dans une logique d'humanisation ${ }^{30}$, et leur regard sur l'immigration reste empreint d'une certaine forme de compassion ethnocentrique. Le proverbe «charité bien ordonnée commence par soi-même» - que les anglophones traduisent par «charity begins to the home » - n'a jamais été aussi vrai dans le contexte actuel caractérisé par un repli identitaire généralisé en France et en Europe : il n'est pas certain que le message avant-gardiste de François soit bien compris. Nul doute que ce pape dérange et qu'il continuera à déranger l'univers catholique pour de nombreuses années encore.

Marseille, le I3 mars 2017

30. Le pape François parle $d^{\prime} \ll$ un chemin constant d'humanisation »: PAPE FRANÇOIS, "Que t'est-il arrivé, Europe humaniste?", art. cité. 